\title{
Running coupling from gluon exchange in the Schrödinger functional
}

\section{Yigal Shamir*}

Raymond and Beverly Sackler School of Physics and Astronomy

Tel-Aviv University, Ramat Aviv, 69978 Israel

E-mail: shamirepost.tau.ac.il

I propose a new method to determine the running coupling in a Schrödinger-functional setup. The method utilizes the scattering amplitude of massless fermions propagating between the time boundaries. Preliminary tests show the statistical fluctuations of the new observable to be about half those of the standard Schrödinger-functional running coupling.

The XXIX International Symposium on Lattice Field Theory - Lattice 2011

July 10-16, 2011

Squaw Valley, Lake Tahoe, California

${ }^{*}$ Speaker. 


\section{Introduction}

There is a growing body of non-QCD lattice work relevant to physics beyond the Standard Model. The vector-like theories under study vary in their number of colors, their number of flavors, and the group representation chosen for the fermions.

At present, the main interest in these models is as candidate technicolor theories. Phenomenological constraints [1] require that a successful technicolor theory have a slowly running coupling constant ("walking technicolor"), as well as a large mass anomalous dimension. A reliable determination of these quantities requires nonperturbative lattice techniques.

The Schrödinger functional (SF) is a finite-volume setup especially suited for this study. By suitably prescribing the spatial components of the gauge field on the time boundaries, a classical, background electric field is induced throughout the four-volume. Measuring the response of the system to changes in the boundary values then allows us to calculate the running coupling $g$ as a function of the system's size $L$.

While providing a de-facto standard method to measure the running coupling, there are reasons to seek alternative methods to determine the running coupling within the SF setup. One reason is that the standard method relies on a very noisy observable, while alternatives might be less so. Also, an alternative method to determine the running coupling will likely have different systematics, thereby providing an important consistency check. This is all the more important because in theories with a slowly running coupling one is forced to use (relatively) strong bare coupling in order to explore the vicinity of a (tentative) infrared fixed point.

Another standard measurement in the SF setup is the extraction of the mass anomalous dimension from the volume dependence of $Z_{P}$, the renormalization constant of the pseudoscalar density. This measurement turns out to be far less noisy than that of the running coupling.

Motivated by this simple observation, I will explore the possibility of extracting the running coupling from fermion correlation functions that are not unrelated to those used in the determination of $Z_{P}$. These correlation functions involve an "ingoing" and an "outgoing" fermionantifermion pair. The ingoing fermions have zero spatial momentum, while the outgoing ones have nonzero momentum. This kinematics enforces the exchange of a gluon between the two fermion lines, and the result is that the correlation function is parametrically of order $g^{2}$.

\section{The Schrödinger functional}

I will briefly describe the lattice implementation of the SF for two flavors of Wilson fermions [2]. It is assumed that the hopping parameter has been tuned to its critical value by enforcing an axial Ward identity, making the fermions massless.

The lattice is a four-dimensional mesh of $N^{4}$ sites, whose linear size is $L=N a$, where $a$ is the lattice spacing. The spatial components $U_{k}=\exp \left(i a A_{k}\right)$ of the $\mathrm{SU}(N)$ gauge field on the time boundaries (at $t=0$ and $t=L$ ) are fixed to have commuting, spatially constant values. Different values are chosen for the two boundary, with the effect that $S_{c l}$, the minimum of the classical action, describes a constant electric field throughout the bulk. In lattice perturbation theory, calculating the 
first quantum correction to the effective action $\Gamma$ gives

$$
\Gamma=\left(\frac{1}{g_{0}^{2}}+\frac{b_{1}}{32 \pi^{2}} \log (L / a)\right) S_{c l}=\frac{1}{g^{2}(L)} S_{c l}
$$

where $g_{0}$ is the bare lattice coupling, and $b_{1}$ is the coefficient of the one-loop beta function. This result is true up to discretization errors, i.e., up to corrections proportional to positive powers of the lattice spacing $a$. The boundary values of $A_{k}$ depend on the dimensionful parameter $L$ only. As a result, $g(L)$ is the one-loop running coupling. If we allow the boundary data to depend in addition on a dimensionless parameter $\eta$, the $\eta$-derivative of the effective action is an observable that can be calculated using standard Monte-Carlo techniques. The SF running coupling is thus defined nonperturbatively as

$$
\frac{K}{g_{S F}^{2}(L)}=\left.\frac{\partial \Gamma}{\partial \eta}\right|_{\eta=0}, \quad K=\left.\frac{\partial S_{c l}}{\partial \eta}\right|_{\eta=0} .
$$

The minimal classical action $S_{c l}$, and hence $K$, depend on $L / a$, but, as it turns out, the discretization errors are of order $a^{4}$, making them practically negligible.

The pseudoscalar renormalization constant $Z_{P}=Z_{P}(L)$ is calculated in the SF setup via [3] $]^{1}$

$$
\begin{aligned}
Z_{P} & =f_{P}(L / 2) / \sqrt{f_{1}}, \\
f_{P}(t) & =\sum_{\vec{x}}\left\langle\bar{\psi}(\vec{x}, t) \gamma_{5} \tau_{a} \psi(\vec{x}, t) \zeta \gamma_{5} \tau_{a} \zeta\right\rangle, \\
f_{1} & =\left\langle\bar{\zeta}^{\prime} \gamma_{5} \tau_{a} \zeta^{\prime} \bar{\zeta} \gamma_{5} \tau_{a} \zeta\right\rangle .
\end{aligned}
$$

The Pauli matrices $\tau_{a}$ act on the isospin index of the fermion field. $\zeta$ and $\bar{\zeta}\left(\zeta^{\prime}\right.$ and $\left.\bar{\zeta}^{\prime}\right)$ are gauge invariant, zero-momentum fermion and antifermion wall sources located near the $t=0(t=L)$ boundaries [3]. The anticipated scaling behavior is

$$
\begin{aligned}
f_{P}(L / 2) & \sim L^{3} Z_{P}(L) Z_{\zeta}^{2}(L), \\
f_{1} & \sim L^{6} Z_{\zeta}^{4}(L),
\end{aligned}
$$

where $Z_{\zeta}$ is the wall-source renormalization factor. The ratio (2.3) is independent of $Z_{\zeta}$, thereby providing an acceptable prescription for $Z_{P}$.

\section{Running coupling from gluon exchange}

Let us turn our attention to the correlation function (2.5) of the four wall sources, used for normalization in the definition of $Z_{P}$. We generalize this correlation function to

$$
f(\vec{n})=\left\langle\bar{\zeta}^{\prime}(-\vec{n}) \gamma_{5} \tau_{a} \zeta^{\prime}(\vec{n}) \bar{\zeta} \gamma_{5} \tau_{a} \zeta\right\rangle
$$

Here we have allowed the wall sources located near the $t=L$ boundary to have nonzero momentum $\vec{p}=(2 \pi / L) \vec{n}$, where the integer-valued vector $\vec{n}$ lives on the reciprocal lattice. The wall sources near the $t=0$ boundary are kept at zero momentum. Let us now consider the ratio

$$
R(\vec{n})=\frac{f(\vec{n})}{f(\overrightarrow{0})}=\frac{f(\vec{n})}{f_{1}}
$$

\footnotetext{
${ }^{1}$ I omit normalization factors conventionally included in these definitions.
} 


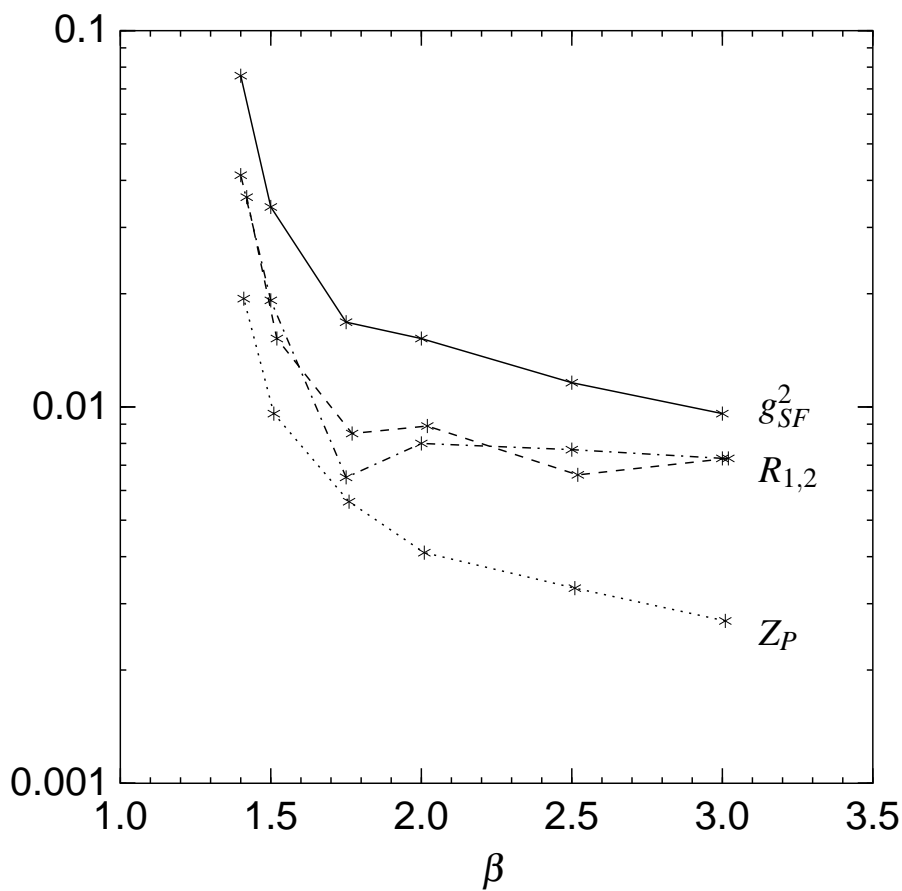

Figure 1: Relative error in several observables, plotted as a function of the bare coupling. Data points for $1 / g_{S F}^{2}, R_{1}, R_{2}$, and $Z_{P}$, are connected by a solid, dashed-dot, dashed, and dotted line respectively. The $R_{2}$ points have been slightly shifted horizontally for better visibility. See text for further explanation.

where we fix the components of $\vec{n}$ at small integers. In the absence of any other infrared scale, the anticipated scaling is

$$
f(\vec{n}) \sim L^{6} Z_{\zeta}^{4}(L) g^{2}(L), \quad \vec{n} \neq \overrightarrow{0} .
$$

In contrast with Eq. (2.7), momentum must now be transferred between the fermion lines. This requires a gluon exchange, which costs a factor of $g^{2}$. In the ratio (3.2), once again, the dependence on $Z_{\zeta}$ cancels out, and the scaling is $R(\vec{n}) \sim g^{2}(L)$. We may thus define a gluon-exchange running coupling via

$$
g_{G E}^{2}(\vec{n} ; L)=\frac{R(\vec{n} ; L)}{K(\vec{n})},
$$

where now $K(\vec{n})$ is the tree-level value of $R(\vec{n} ; L)$ in the limit $L / a \rightarrow \infty$. Each momentum vector $\vec{n}$ amounts to a different scheme. Standard arguments imply that $g_{S F}^{2}(L)$ as well as $g_{G E}^{2}(\vec{n} ; L)$, for all $\vec{n}$, have the same two-loop beta function.

A first look at the new observables is offered by Fig. 1. This is an SU(2) gauge theory, with two Dirac fermions in the adjoint representation [4. 5]. An ensemble of 8000 configurations of volume $8^{4}$ was generated for each of the six bare-coupling values $\beta=3.0,2.5,2.0,1.75,1.5$ and 1.4. The lattice implementation is the same as in Ref. [5], where also the values of $\kappa_{c}(\beta)$ may be found. Fig. 1 shows the relative error in several observables. Two of them are the familiar $1 / g_{S F}^{2}$ and $Z_{P}$. The other two, $R_{1}$ and $R_{2}$, are linear combinations of $R(\vec{n})$ (Eq. (3.2)), for momentum vectors whose components are either $2 \pi / L$ or zero. $R_{1}$ sums up $R(\vec{n})$ for the three possibilities where a single component of $\vec{n}$ is nonzero. $R_{2}$ is similarly constructed, except that two momentum components are nonzero. 
As can be seen from the figure, the relative error in $Z_{P}$ is roughly one fourth that of $1 / g_{S F}^{2}$, while the relative errors in $R_{1}$ and $R_{2}$ are roughly in the middle. This very preliminary result suggests that the new observables indeed have smaller statistical fluctuations than those of $1 / g_{S F}^{2}$.

\section{Discussion}

In order to make use of the newly defined gluon-exchange coupling it will be necessary to compute the relevant tree-level amplitudes, $K(\vec{n})$, that enter as normalization constants in the definition (3.4). Each $K(\vec{n})$ is the infinite-volume limit of the corresponding finite-volume tree-level amplitudes. A calculation of the tree-level amplitudes at finite $L$ will also provide important information about the discretization errors in the new observables. Unlike in the very special case of $1 / g_{S F}^{2}$, where, as mentioned above, tree-level discretization errors go like $a^{4}$, for the gluonexchange coupling the discretization errors could be of order $a^{2}$ or even of order $a$. It should be noted that, in the event that discretization errors turn out to be relatively big for individual $R(\vec{n})$ amplitudes, one may attempt to construct linear combinations of these amplitudes that have smaller (tree level) discretization errors. The question will then be whether this can be done without degrading the statistical quality of the observable. Work on these questions is underway.

\section{Acknowledgments}

I thank Tom DeGrand, Stefan Sint, Rainer Sommer, and Ben Svetitsky for discussions and useful suggestions. This research is supported by the Israel Science Foundation under grant no. $423 / 09$.

\section{References}

[1] For a review, see for example C. T. Hill and E. H. Simmons, Phys. Rept. 381, 235-402 (2003) [hep-ph/0203079].

[2] M. Lüscher, R. Narayanan, P. Weisz and U. Wolff, Nucl. Phys. B 384, 168 (1992) [hep-lat/9207009]. M. Lüscher, R. Sommer, P. Weisz and U. Wolff, Nucl. Phys. B 413, 481 (1994) [hep-lat/9309005]. M. Della Morte, R. Frezzotti, J. Heitger, J. Rolf, R. Sommer and U. Wolff [ALPHA Collaboration], Nucl. Phys. B 713, 378 (2005) [hep-lat/0411025].

[3] S. Sint and P. Weisz [ALPHA collaboration], Nucl. Phys. B 545, 529 (1999) [hep-lat/9808013]. S. Capitani, M. Lüscher, R. Sommer and H. Wittig [ALPHA Collaboration], Nucl. Phys. B 544, 669 (1999) [hep-lat/9810063]. M. Della Morte, R. Hoffmann, F. Knechtli, J. Rolf, R. Sommer, I. Wetzorke and U. Wolff [ALPHA Collaboration], Nucl. Phys. B 729, 117 (2005) [hep-lat/0507035].

[4] F. Bursa, L. Del Debbio, L. Keegan, C. Pica and T. Pickup, Phys. Rev. D 81, 014505 (2010) [arXiv:0910.4535 [hep-ph]].

[5] T. DeGrand, Y. Shamir and B. Svetitsky, Phys. Rev. D 83, 074507 (2011) [arXiv:1102.2843 [hep-lat]]. 